\title{
IR thermal imaging solutions for flight applications
}

\author{
Dipl.-Ing. Manfred Pfadt \\ ${ }^{1}$ MICRO-EPSILON MESSTECHNIK GmbH \& CO. KG
}

Königbacher Str. 15 / 94496 Ortenburg / Germany

Manfred.Pfadt@micro-epsilon.de

\begin{abstract}
IR thermal imaging solutions for flight applications:
Thermal imagers detect and measure temperatures on any surface. Based on this, the actual camera technology will be explained from physics and the background technology. In addition practical solutions based on IR imaging technologies for R\&D and in-flight applications will be illustrated. In the final outlook possible further uses for the technology will be discussed i.e. non-destructive material analysis.
\end{abstract}

Key words: thermal imaging, IR camera, infrared camera, flight thermography, thermolMAGER

\begin{abstract}
About Micro-Epsilon [1]
Micro-Epsilon is a medium-sized, family-run technology company based in Lower Bavaria. The sensor specialist provides technical high performance, customized solutions for industrial measurement and inspection tasks: from automotive and semiconductor manufacturing to metal processing. The company's portfolio includes IR temperature sensors and colour recognition systems, sensors for distance and displacement measurement, as well as dimensional measurement and defect recognition systems. Starting out as a small company but with big ideas, 45 years later this German enterprise has become an international group of companies. Today, Micro-Epsilon employs more than 600 people worldwide, with each one of these people contributing to the company's success. Continuous development efforts, extensive know-how and a wide cooperation network enable the creation of innovative, high precision products.
\end{abstract}

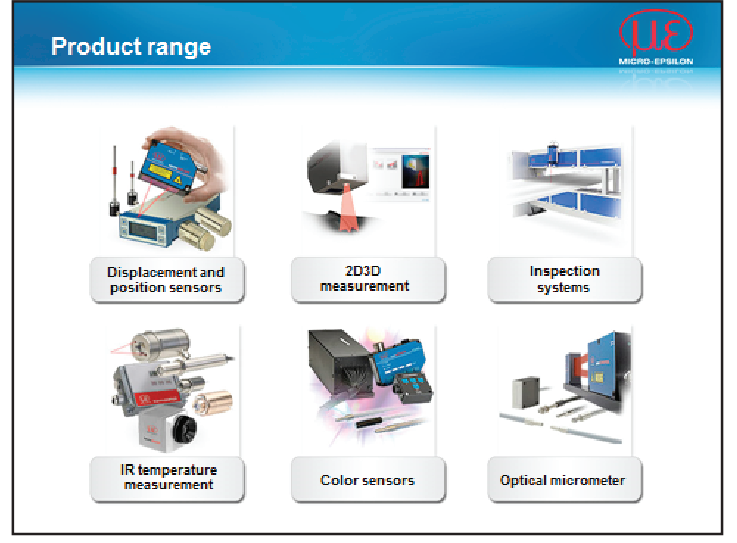

Fig. 1. Overview product range

\section{Principle of infrared temperature} measurement [2]

Infrared radiation is emitted from everybody whose temperature is above absolute zero. The infrared sensor captures the emitted radiation and deflects this to one or more detectors. The energy of the infrared radiation is converted into 
electrical signals in the detector, which are then converted into temperature values based on the calibration of the sensor and the specified emissivity.

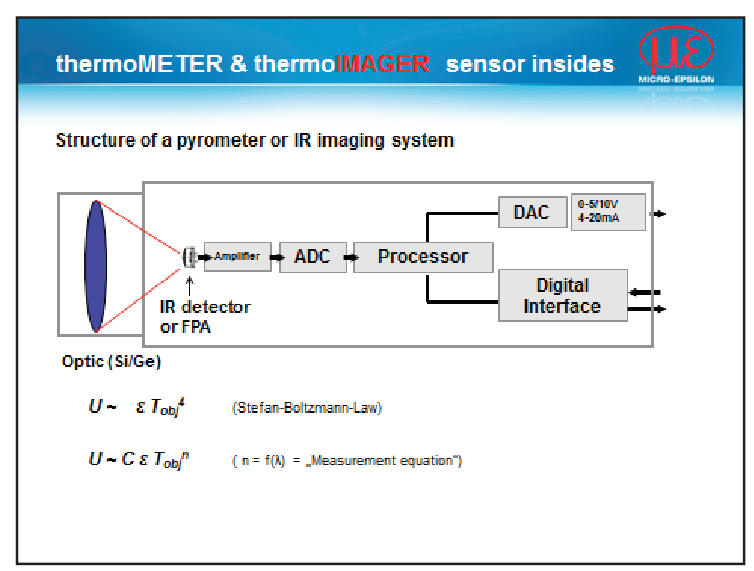

Fig. 2. Structure of an IR system

Based on this evaluation, the measured temperature can be shown on a display, output as an analogue signal or displayed on a PC via a digital output. All bodies emit infrared radiation in three different ways. They can emit radiation, reflect it from the surroundings or transmit it through itself. How the individual factors interact depends on the material of the measurement object. However, only the emitted radiation is important for measurements. The relationship of the individual emissions to each other is described by the emissivity. If it is assumed for solid bodies that they let through zero radiation, the transmission can be replaced with zero. The emissivity is still only composed of emission and reflection. Therefore, it is now easily to understand why objects such as polished and shiny metals can only have a low emission, as radiation from the surroundings is strongly reflected by these surfaces. In contrast, objects such as clothing or matt black surfaces reflect very little and so are well suited to non-contact temperature measurement.

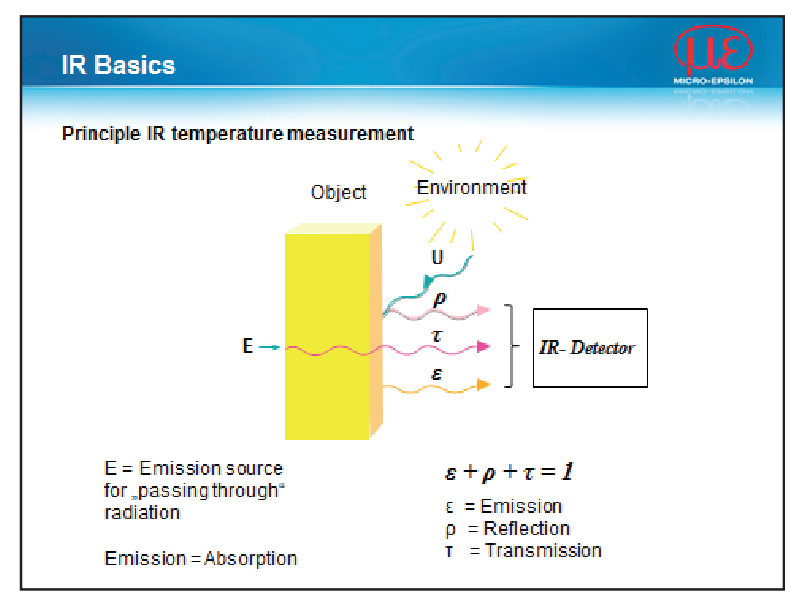

Fig. 3. IR measurement (influencing factors)

The 350-gramme thermolMAGER TIM LightWeight thermal imaging camera can be mounted to a quadcopter to carry out defect analysis i.e. on solar cells.

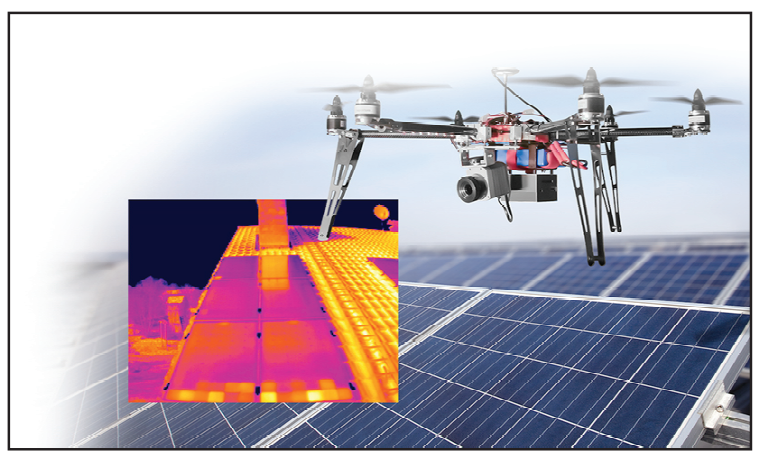

Fig. 4. TIM LightWeigt on a multicopter [3]

Defective solar cells can destroy an entire module. Thermography is therefore a great method of preventative maintenance on photovoltaic installations: any noticeable differences in temperature can be used to reliably detect electrical, mechanical, installation and processing-related defects, including short circuits, inactive cells, moisture, and poorly soldered joints. As part of scheduled maintenance operations, thermography can provide valuable information for resolving warranty claims.

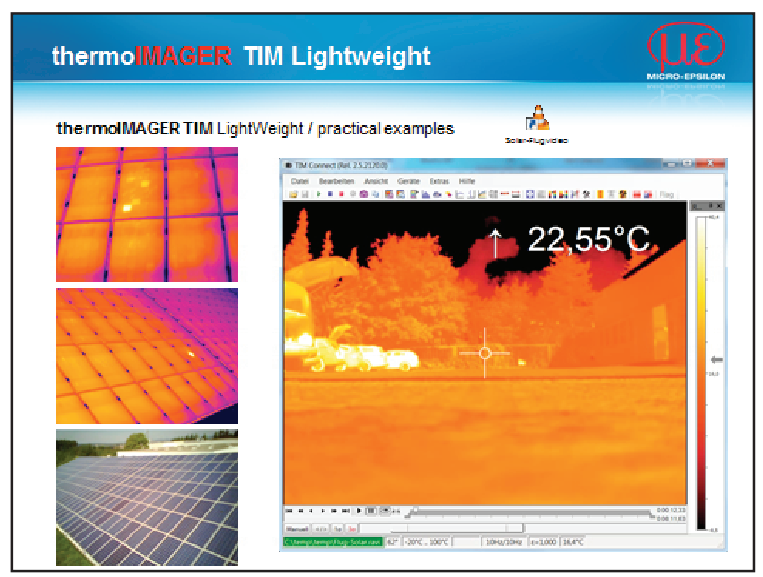

Fig. 5. TIM LightWeigt inflight photos

Inspections using infrared cameras are performed in a non-contact, non-destructive manner from a safe distance. The new miniature thermoIMAGER TIM LightWeight IR camera from Micro-Epsilon is for use on a quadcopter (similar to a small, remotecontrolled helicopter with 4 blades) ideal. Design factors to consider when using an IR 
camera as part of flight operations include low weight, autonomous control and sufficiently high camera resolution to ensure high quality IR images.

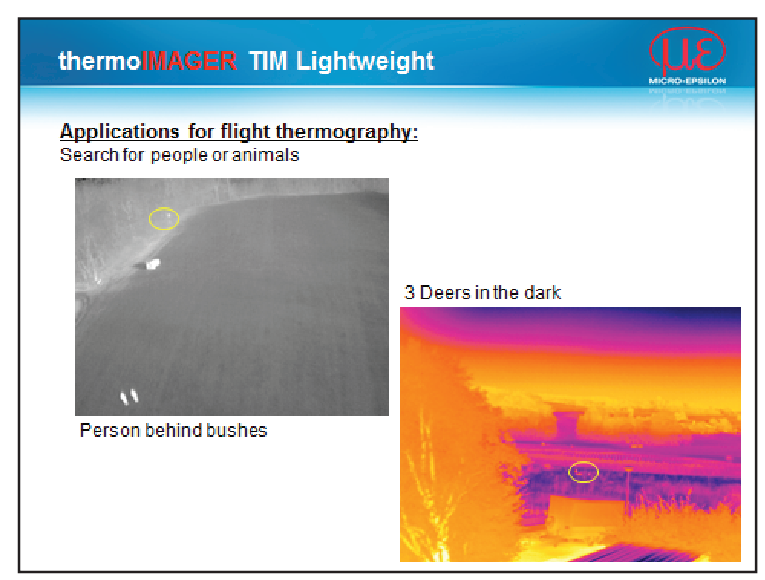

Fig. 6. TIM LightWeigt inflight photos

The new system consists of a miniature IR camera and the NetBox mini PC. With a total weight of just 350 grams, the TIM LightWeight is the perfect choice for flight applications. IR videos can be launched directly through a button on the camera housing; the recording is stored on a microSD storage card in NetBox. The high resolution infrared camera offers an optical resolution of $382 \times 288$ pixels, with a thermal resolution of up to $40 \mathrm{mK}$

The world's smallest VGA infrared camera, the thermolMAGER TIM 640, extends the MicroEpsilon range of industrial infrared cameras. The thermolMAGER TIM 640 performs radiometric imaging and video recording at a resolution of $640 \times 480$ pixels, detecting even the smallest of thermal defects reliably.

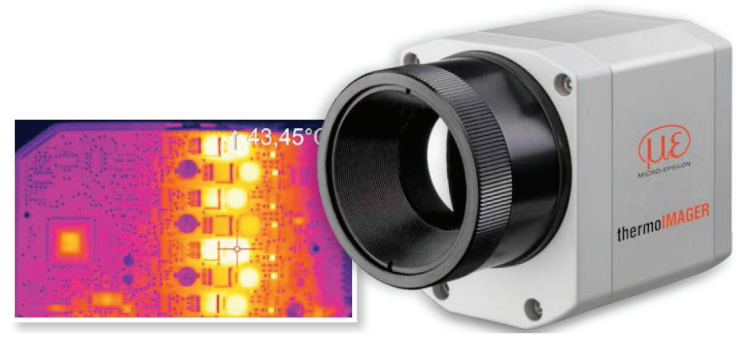

Fig. 7. TIM 640 (System and IR image) [4]

With dimensions of $46 \times 56 \times 90 \mathrm{~mm}$ and weighing just $320 \mathrm{~g}$, the thermoIMAGER TIM 640 stands out due to its high optical resolution at an image frequency of $32 \mathrm{~Hz}$. A process interface with analogue and digital $\mathrm{I} / \mathrm{O}$ simplifies process integration. The scope of supply includes the TIM Connect evaluation software. The temperature range is from $-20^{\circ} \mathrm{C}$ to $+900^{\circ} \mathrm{C}$, the thermal sensitivity is $75 \mathrm{mK}$.

Outstanding features such as high resolution, compactness, and user-friendly integration make the VGA infrared camera, the thermoIMAGER TIM 640, suitable for applications in quality control, process monitoring and analysing electronic microcomponents in almost every industrial sector. Industry-specific software enables the monitoring of very small components during production (inline thermography), especially in plastics injection moulding processes.

\section{In-line thermography for security sensible parts:}

The pressure on quality and cost in plastics processing and in particular injection moulding means that in-line inspection of product quality is increasingly important. As cycle times continue to reduce and process windows become ever tighter, in-line control is becoming more critical. The developed system monitors the process and ensures $100 \%$ quality control in the production line.

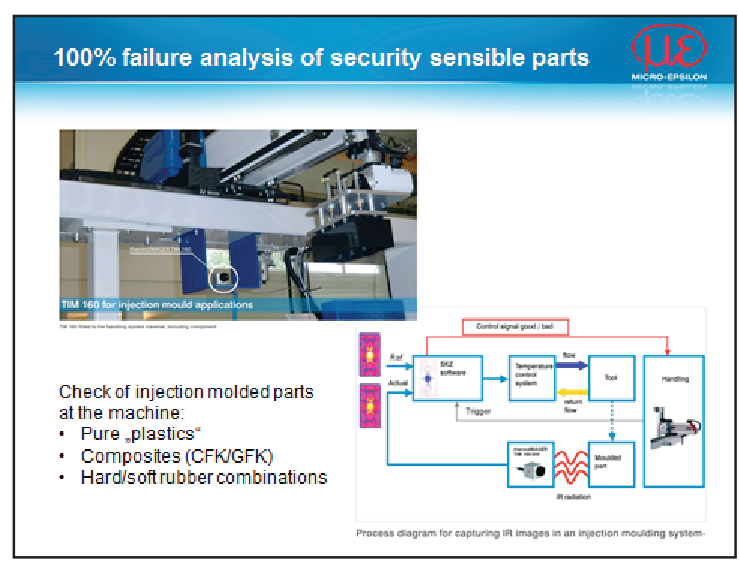

Fig. 8. 100\% Inline process control [5]

Modern injection moulding systems are usually equipped with an automatic handling system for removal and storage of components. These optimise the positioning of a component in front of a thermal imaging camera. With this, a precise time window for recording thermographic images can also be adhered to. These two points ensure comparability of the thermographic images from one video image to another. During automatic removal of the newly produced components, a good/bad component decision must be made within a short time frame. The aim here is to prevent problems that disrupt further processing and to take corrective measures as quickly as possible. As the exact position of the defects is not known, an infrared thermography image that screens the component is suitable for complete analysis. Furthermore, visual inspection systems often cannot detect incorrect temperature control or hidden defects (e.g. a missing bridge).

Together with the SKZ (Süddeutsches Kunststoff Zentrum) in Würzburg, Micro-Epsilon 
has developed a thermography-based solution for the plastics industry. The in-line thermography system detects variations in quality by using an infrared camera. This records the entire component in up to six different views and examines it.

The principle is simple: a thermolMAGER TIM160 TIM400 or TIM640 miniature thermal imaging camera records the infrared radiation emanating from the work piece and visualises it. The temperature distribution provides a global quality statement about incorrect temperature control of the work piece, malfunction of the tool, visible geometry errors and hidden defects. As the first 'good' part is detected reliably immediately after restart, the system minimises start-up rejects following periods of downtime or tool replacement. Evaluation occurs during idle times between two image shots. The handling system presents the newly injected component to the thermoIMAGER TIM from one or more sides. The camera records an IR image in synch with the handling system.

The application specific software, communicates with the control system, the handling system and the thermoIMAGER TIM measurement system. The software compares the infrared images associated with the component to stored references. The software displays the reference image, the IR image of the component and the difference between the two images. The identified temperature differences provide the basis for a good/bad decision. The visual presentation provides the operator with a quick process overview. Temperature alarm limits (local, global and relative) can be freely specified using the operating software. Defective components can be sorted and removed depending on these settings. Furthermore, the operator can be warned of any errors, for example by an indicator display, or in extreme cases, the machine can be switched off. The limit values for different injection moulded parts can be stored in a component database and retrieved from there. In addition to the immediate sorting of components, an IR image of each shot is stored on the hard drive or network drive. This helps to provide $100 \%$ traceability of components, which is often required.

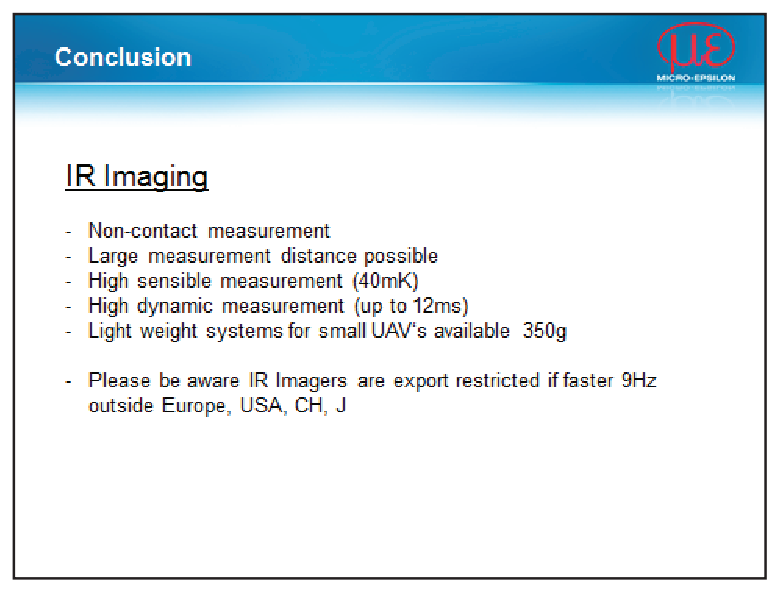

Fig. 9. Advantages of IR imaging systems

\section{References}

[1] http://www.micro-epsilon.com

[2] http://www.microepsilon.com/download/products/dat--infraredbasics--en.pdf

[3] http://www.micro-epsilon.com/temperaturesensors/thermolMAGER/thermoIMAGER_TIM_Li ghtweight/index.html

[4] http://www.micro-epsilon.de/temperaturesensors/thermolMAGER/thermoIMAGER_640/ind ex.html

[5] http://www.microepsilon.de/press/publication/pub-de--2014-06-Verraeterisches_Waermebild.pdf 